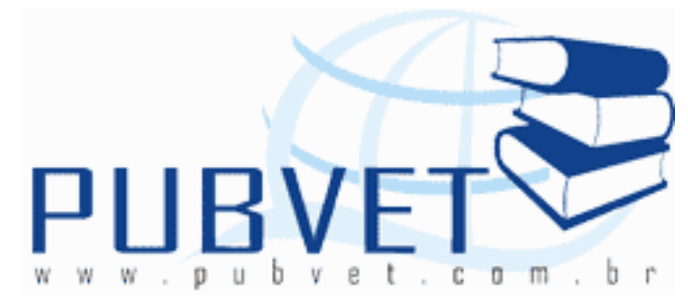

PUBVET, Publicações em Medicina Veterinária e Zootecnia.

Disponível em: <https://doi.org/10.31533/pubvet.v02n11a457>.

\title{
Leiomioma vaginal em cadela associado à compressão de cólon - relato de caso
}

Bernardo Fernandes Lopes ${ }^{1}$, Adrienne Bretas Lanis ${ }^{1}$, Priscila Araújo Lyra

Guedes de Morais², Evandro Zacché Pereira², Daniel Capucho de Oliveira², Germana Marchetti da Silva³, Letícia Binda Baungarten³, Lousiane de Carvalho Nunes ${ }^{4}$, Lenir Cardoso Porfírio ${ }^{4}$, Patrícia Maria Coletto de Freitas ${ }^{4}$, Marcelo Resende Luz ${ }^{4}$, Fabiano Séllos Costa ${ }^{4}$

1-Médico veterinário autônomo, DIAGNOPET Vitória- ES.

2-Acadêmico de Medicina Veterinária, CCA-UFES, Bolsista de Iniciação Científica do Programa PIVIC-PETROBRÁS-UFES.

3-Médico Veterinário Residente, CCA-UFES, Alegre-ES.

4-Professor Adjunto de Diagnóstico por Imagem e Clínica Médica de Pequenos Animais- Centro de Ciências Agrárias da Universidade Federal do Espírito Santo - ES.

\section{RESUMO}

Os leiomiomas e fibromas são as neoplasias mais descritas encontradas na vagina e vulva de cadelas idosas, porém raramente causando compressão de cólon e levando ao tenesmo em cães. O diagnóstico definitivo é realizado através de exame histopatológico. O exame ultra-sonográfico pode ser útil 
para detectar massa abdominal, além de orientar o direcionamento dos demais exames complementares e indicação do tratamento cirúrgico do paciente. Relata-se neste trabalho o caso de uma cadela que se apresentava ao exame ultra-sonográfico uma massa mensurando aproximadamente $7,2 \mathrm{~cm}$ em seu maior diâmetro e localizada caudo-ventralmente à bexiga, promovendo uma compressão e deslocamento dorsal em cólon descendente. Após análise histopatológica foi confirmado o diagnóstico de leiomioma vaginal como a causa primária da compressão de cólon neste caso.

Palavras - chave: Leiomioma, tenesmo, ultra-sonografia, cães.

\section{Vaginal leiomyoma in a bitch associated with compression of colon - case report}

\section{ABSTRACT}

Leyomiomas and fibromas are the most described neoplasms found in old bitches' vagina and vulva, but they rarely cause colon compression leading to tenesm in dogs. The definitive diagnosis is realized with the histopathologic exam. The ultrasonography can be useful to detect abdominal masses, and can also direct the others complementary examinations and the indication of surgical treatment. It is reported in this paper the case of a bitch that, in the ultrasonographic examination, presented a mass measuring approximately 7,2 $\mathrm{cm}$ in its major diameter and localized caudoventrally to the bladder, promoting a compression and a dorsal displacement in the descendent colon. After histopathological analysis it was confirmed the diagnosis of vaginal leyomioma as the primary cause of this case of colon compression.

Keywords: Leyomioma, tenesm, ultrasound, dogs 


\section{INTRODUÇÃO}

Enfermidades urogenitais tais como prostatites, abcessos prostáticos, cistites, vaginites e litíase uretral, podem promover tenesmo fecal e disquezia em cães. Processos neoplásicos da uretra, bexiga, próstata ou vagina também podem desencadear esta sintomatologia clínica, entretanto, sua ocorrência é considerada incomum (KATAMOTO, 2003).

Os tumores da vagina e da vulva são a segunda neoplasia mais comum do trato reprodutivo das cadelas (HERRON, 1983); segundo THACHER \& BRADLEY, (1983) representam aproximadamente $3 \%$ de todas as neoplasias em cães. As neoplasias mais descritas são os leiomiomas e os fibromas (KYDD, 1986).

Os leiomiomas são neoplasias de crescimento lento e tem caráter não invasivo e não metastático. Freqüentemente esta enfermidade está associada à ausência de sinais clínicos nos animais acometidos (KLEIN, 2007 e JOHNSTON et al., 2001). Sua ocorrência em cães foi descrita em diversas regiões do corpo nas quais o músculo liso está presente, tais como vagina, útero e intestinos (KATAMOTO, 2003).

Segundo a literatura o leiomioma vaginal ocorre com maior freqüência em cadelas nulíparas e pode estar associado a irregularidades no ciclo estral ou a pseudociese (SILVA \& VARASCHIN, 1996). Apresenta alta incidência em cadelas idosas não castradas (KLEIN, 2007). Em estudos apresentados por THACHER et. al., (1983); e KYDD et. al. (1986) os autores indicam que a ocorrência desta neoplasia parece ser hormônio-dependente, uma vez que nenhuma das cadelas diagnosticadas com leiomioma, fibroma ou tumores polipóides era castrada ao momento do diagnóstico.

A avaliação radiográfica e ultra-sonográfica da região abdominal podem confirmar a presença de massas abdominais. O exame histopatológico é necessário para o diagnóstico definitivo (KLEIN, 2007). O diagnóstico ultrasonográfico pode ser um método suficiente para o direcionamento dos demais exames complementares e indicação do tratamento cirúrgico do paciente. 
O tratamento destes tumores é realizado através de remoção cirúrgica da massa e realização de histerectomia (KLEIN, 2007). Estudos mostraram que ocorreram recidivas em $15 \%$ das cadelas nas quais a histerectomia não foi realizada após remoção da massa (THACHER et. al., 1983) e (KYDD et. al., 1986).

\section{RELATO DE CASO}

Relata-se o caso de uma cadela rottweiler, castrada, 13 anos, encaminhada para o Serviço de Diagnóstico por Imagem do Hospital Veterinário da Universidade Federal do Espírito Santo. O animal apresentavase com histórico de dor durante a defecação e fezes em formato achatado com evolução de 2 meses, sendo que, segundo o proprietário, o animal não defecava há aproximadamente uma semana.

Ao exame ultra-sonográfico da cavidade abdominal observou-se a presença de uma massa mensurando aproximadamente $7,2 \mathrm{~cm}$ em seu maior diâmetro e localizada caudo-ventralmente à bexiga, promovendo uma compressão e deslocamento dorsal em cólon descendente. A massa apresentava-se com uma ecotextura discretamente heterogênea, não sendo observado lojas císticas ou áreas de calcificação.

Com base nos achados clínicos e ultra-sonográficos foi indicada realização de citologia aspirativa da massa guiada por ultra-som, entretanto, não foi possível concluir o diagnóstico. Sendo assim o animal foi encaminhado para retirada cirúrgica da massa e posterior análise histopatológica que revelou presença de massa neoplasica encapsulada, localizada em região da vagina na qual as células eram fusiformes, arranjadas em fascículos. Estes achados foram compatíveis com leiomioma vaginal. 


\section{DISCUSSÃO E CONCLUSÃO}

Conforme citado na literatura consultada, o leiomioma vaginal é mais freqüente em cadelas não castradas, sendo considerado raro em cadelas castradas. Nosso relato demonstra que apesar de apresentar rara incidência, não devemos excluir a possibilidade da ocorrência de leiomioma vaginal em cadelas castradas.

Ressalta-se o achado incomum desta neoplasia como causa primária de tenesmo fecal na espécie canina, uma vez que as causas mais freqüentes segundo a literatura estariam relacionadas a doenças prostáticas, tais como prostatites e abcessos prostáticos.

Destaca-se também a importância do exame ultra-sonográfico neste caso, propiciando uma visualização direta da massa neoplásica e permitindo o direcionamento dos demais exames complementares realizados e o adequado tratamento do paciente.

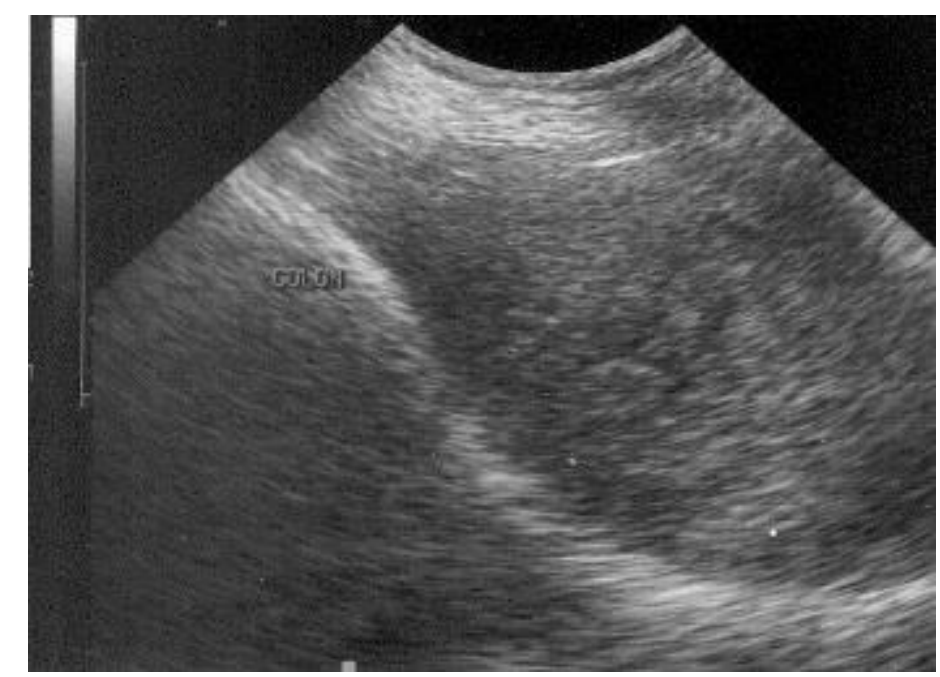

Figura 1- Avaliação ultra-sonográfica de leiomioma vaginal promovendo compressão de cólon em cadela. 


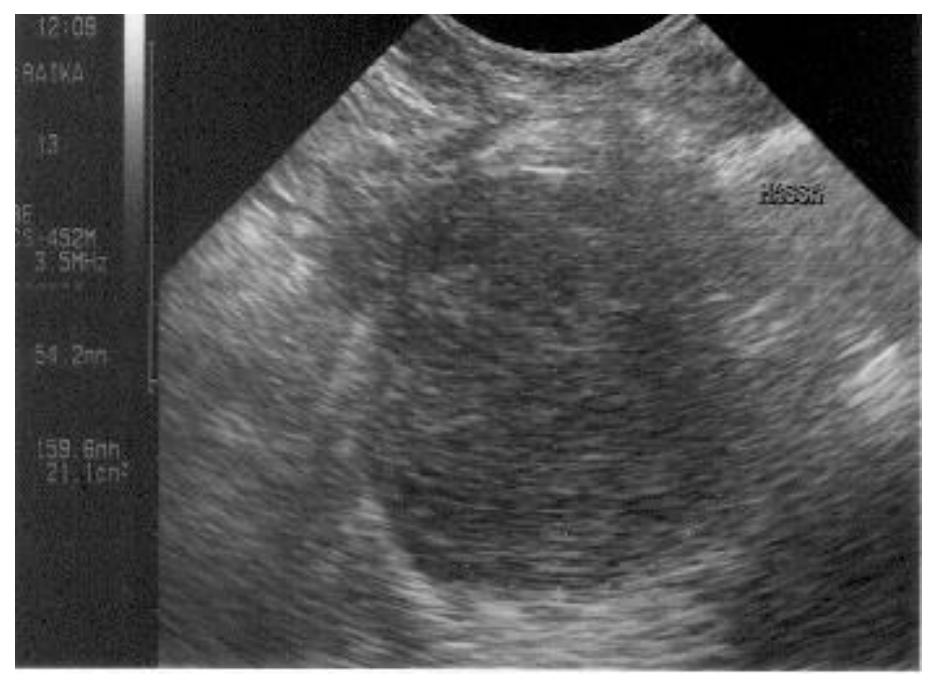

Figura 2- Ultra-sonografia da massa abdominal mensurando aproximadamente $5,42 \mathrm{~cm}$ em seu maior diâmetro ao corte transversal.

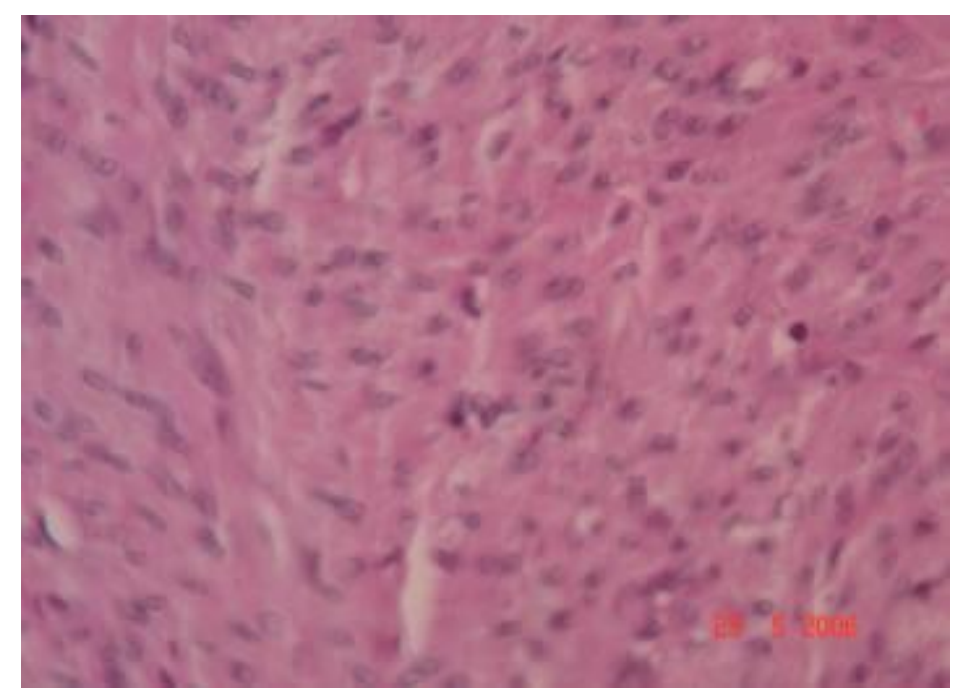

Figura 3- Fotomicrografia de leiomioma vaginal em cadela da raça Rottweiler (Hematoxilina e Eosina, aumento de 400 vezes). 
Lopes, B.A., Lanis, A.B., Morais, P.A.G. et al. Leiomioma vaginal em cadela associado à compressão de cólon - relato de caso. PUBVET, Londrina, V. 2, N. 47, Art\#457, Nov4, 2008.

\section{REFERÊNCIAS}

1. HERRON, M. A. Tumors of the canine genital system. J Am Anim Hosp Assoc. v. 19, p. 981-994, 1983.

2. JOHNSTON, D. S.; KUSTRIZ, R. V. M.; OLSON, P. N. S. Canine and feline Theriogenology. Philadelphia: Saunders, p.463-472, 2001.

3. JUNQUEIRA, L. J.; CARNEIRO, J. Histologia Básica. Editora Guanabara Koogan. SA., Rio de Janeiro, 8 ed., p. 382-383, 1995.

4. KATAMOTO, H.; KUMAGAI, D.; KOUZAI, N.; TAKIGAMI, S.; KUAMURA, M.; YAMATE, J.; KOTANI, T. Space-occupying leiomyoma in the pelvic canal of a dog. Journal of Small Animal Practice. v. 44, p. 277-279, 2003.

5. KLEIN, M.K., Tumors of female reproductive system in:WITHROW, S. J.; MACEWEN, E. G. Small Animal Clinical Oncology. WB Saunders. St.Louis, 4 ed., p. 610-618, 2007.

6. KYDD, D. M.; BURNIE, A. G. Vaginal neoplasia in the bitch: A review of 40 cases. Journal of Small Animal Practice. v. 27, p. 255-263, 1986.

7. SILVA, A.M.; VARASCHIN, M.S. Leiomioma vaginal em cadela. A Hora Veterinária. v. 15, n. 90, 1996.

8. THACHER, C.; BRADLEY, R.L. Vulvar and vaginal tumors in the dog: A retrospective study. Journal of American Veterinary Association. v. 183, n. 6, p. 690- 692, 1983. 